\title{
Review of: "Predictors of hypoxemia in type-B acute aortic syndrome: a retrospective study"
}

\author{
Zhongheng Zhang ${ }^{1}$ \\ 1 Zhejiang University
}

Potential competing interests: The author(s) declared that no potential competing interests exist.

The study explored risk factors of postoperative hypoxia in patients with acute aortic syndrome (AAS), as well as its impact on the clinical outcomes. While there has been many studies exploring the same issue in the literature ${ }^{[1][2]}$, this study confirmed previous findings by using a single center data. however, some new biomarkers are identified in the current study, which requires further validation in multicenter trials.

\section{References}

1. ^Huiqing Ge, Ye Jiang, Qijun Jin, Linjun Wan, et al. (2018). Nomogram for the prediction of postoperative hypoxemia in patients with acute aortic dissection. BMC Anesthesiol, vol. 18 (1). doi:10.1186/s12871018-0612-7.

2. `Yinghua Wang, Song Xue, Hongsheng Zhu. (2013). Risk factors for postoperative hypoxemia in patients undergoing Stanford A aortic dissection surgery. J Cardiothorac Surg, vol. 8 (1). doi:10.1186/1749-8090-8-118. 